\title{
Análise da eficiência das regiões administrativas do Rio de Janeiro em função da mobilidade
}

\section{Clara Maia Bevilaqua Contursi ${ }^{1}$, Raquel Silva de Oliveira ${ }^{2}$, Marcelino Aurélio Vieira da Silva ${ }^{3}$, Licínio da Silva Portugal ${ }^{4}$}

\author{
${ }^{1}$ Programa de Engenharia de Transportes - PET/COPPE/UFRJ, Brasil, claracontursi@pet.coppe.ufrj.br \\ 2Programa de Engenharia de Transportes - PET/COPPE/UFRJ, Brasil, raqueloliveira@pet.coppe.ufrj.br \\ 3Programa de Engenharia de Transportes - PET/COPPE/UFRJ, Brasil, marcelino@pet.coppe.ufrj.br \\ 4Programa de Engenharia de Transportes - PET/COPPE/UFRJ, Brasil, licinio@pet.coppe.ufrj.br
}

\section{Recebido: \\ 15 de março de 2018 \\ Aceito para publicação: \\ 27 de maio de 2018 \\ Publicado: \\ 4 de novembro de 2018 \\ Editor de área: \\ Bruno Vieira Bertoncini}

\section{Palavras-chaves:}

Mobilidade Urbana;

Eficiência;

Análise Envoltória de Dados.

\section{Keywords:}

Urban Mobility;

Efficiency;

Data Envelopment Analysis.

DOI:10.14295/transportes.v26i3.1627

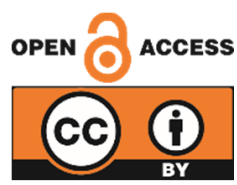

\begin{abstract}
RESUMO
No intuito de contribuir com as discussões acerca da qualidade dos sistemas de transporte no município do Rio de Janeiro, o presente artigo investiga a eficiência de 24 das suas mais relevantes Regiões Administrativas em função da mobilidade. Essa investigação realiza-se por meio da Análise Envoltória de Dados (DEA), a qual estima a eficiência padrão de cada região, e por meio da Avaliação Cruzada, que ranqueia essas eficiências, bem como baseia-se no desempenho de dois indicadores: um de avaliação da eficiência social e outro de eficiência econômica das regiões. Esses indicadores mostram desempenho similar na DEA e bem diferentes na Avaliação Cruzada, evidenciando a discrepância das duas abordagens. Algumas regiões apresentam resultados discordantes (alta eficiência na DEA e baixa na Avaliação Cruzada), como Portuária e Realengo, e outras apresentam resultados equivalentes, como Centro, com alta eficiência, e Santa Cruz, com baixa, tanto na DEA quando na Avaliação Cruzada.
\end{abstract}

\begin{abstract}
In order to contribute to discussions about the quality of transportation systems in the city of Rio de Janeiro, this article investigates, due to mobility, the efficiency of 24 of the most important Administrative Regions, through Data Envelopment Analysis (DEA) which estimates the standard efficiency - and through Cross-Evaluation - which ranks these efficiencies. The analysis is also based on the performance of two indicators: one for the evaluation of social efficiency and another for the economic efficiency. These indicators demonstrated a similar performance in DEA and very different performance in Cross-Evaluation, indicating divergent approaches. Some DMU present different results (high efficiency in DEA and low efficiency in Cross-Evaluation), for example, Portuária and Realengo, and others present equivalent results, for example, Centro present high efficiency and Santa Cruz present low efficiency in DEA and Cross-Evaluation.
\end{abstract}

\section{INTRODUÇÃO}

As cidades são o motor da inovação e do crescimento econômico. Os serviços e empregos, na maioria das vezes, são ofertados nos grandes centros urbanos, que tendem a se valorizar e passar por processos de expansão e periferização que, nos países em desenvolvimento, costumam ocorrer de forma desordenada e sem um planejamento territorial e de transportes sintonizado com o interesse público. Estas localidades periféricas normalmente acolhem as necessidades de moradia dos segmentos da população de menor poder aquisitivo, que precisam diariamente realizar longos e demorados deslocamentos para desenvolverem suas atividades desejadas e disponíveis nas áreas mais centrais. A falta de equidade na distribuição espacial do uso do solo e de transporte público qualificado e integrado reflete condições precárias de acessibilidade 
que causam sérios problemas de mobilidade vivenciados por esta comunidade (Cervero, 2013), como se observa no município do Rio de Janeiro (Mello, 2015).

$\mathrm{Na}$ cidade do Rio de Janeiro - a ser estudada neste artigo - a rede de transportes coletivos de passageiros compreende os sistemas de trens, metrô, barcas, ônibus e vans regulares. No entanto, atualmente, $71 \%$ de todas as viagens realizadas em transporte coletivo são feitas por ônibus (PDTU, 2013). Ainda, segundo o PDTU (2013), há na cidade do Rio de Janeiro, uma predominância de corredores radiais concêntricos, que funcionam como ligação entre a periferia e o centro metropolitano. Se a configuração radial da rede tende a concentrar viagens nos corredores, o que poderia favorecer as modalidades de maior capacidade destinadas a atender grandes demandas de viagens de médio a longo percurso, isto não ocorre no Rio de Janeiro: o trem, o metrô e as barcas não chegam a atender 10\% destes deslocamentos motorizados. Tal divisão modal expressa uma mobilidade problemática fruto de condições de acessibilidade - derivadas da rede estruturante de transporte público - que não valorizam as modalidades mais produtivas socialmente e ambientalmente. 0 que também ocorre no âmbito local no que se refere à mobilidade não motorizada (Portugal, Flórez e Silva, 2010).

Na medida em que este cenário se estende a outros fatores que interferem na mobilidade e se reproduz no âmbito metropolitano, mas também no interior da cidade do Rio de Janeiro entre as suas Regiões Administrativas, este trabalho busca suprir o desconhecimento quanto aos níveis de eficiência associados à mobilidade urbana dessas regiões. Para tal, assume-se como mobilidade "a capacidade dos indivíduos se moverem de um lugar a outro dependendo das suas características socioeconômicas e do desempenho do sistema de transporte” (Tagore e Sikdar, 1995); motivada pela vontade de realizar uma dada atividade; e como eficiência, "a comparação do que foi produzido, dado os recursos disponíveis, com o que poderia ter sido produzido com os mesmos recursos" (Mello et al., 2005). Ambos os conceitos agora mencionados, são melhores desenvolvidos em outras seções deste trabalho.

Observou-se também que o uso de indicadores de mobilidade sustentável no Brasil é incipiente (Costa, 2008), prevalecendo a utilização de indicadores tradicionais e isolados como número de viagens, o que se confirma em revisão feita por Pedro, Da Silva e Portugal (2017).

Nesse contexto, que envolve uma certa complexidade e se caracteriza pela multiplicidade de atores e interesses potencialmente conflitantes, a bibliografia consultada estabelece que os índices ou indicadores, quando devidamente selecionados e usados de forma fundamentada, se configuram em excelentes ferramentas para o diagnóstico dos padrões de mobilidade, das condições de acessibilidade e do monitoramento do impacto de políticas públicas, o que permite acompanhar a evolução de determinados fenômenos e ações (Costa, 2008; Jones, 2014; Mori e Yamashita, 2015; Gonzáles Villada, 2016). Essas ferramentas têm sido utilizadas nos processos de planejamento urbano, principalmente voltados à mobilidade com foco na sustentabilidade (Portugal, 2017).

No entanto, ainda que os indicadores de mobilidade sustentável já sejam explorados e aplicados, há um hiato entre o conhecimento e a aplicação das técnicas utilizadas para a produção desses índices e indicadores (Hull, Silva e Bertolini, 2012; Bertolini, 2017). Este hiato pode se expressar pela determinação de indicadores e índices por meio de abordagens mais subjetivas, não necessariamente amparadas e complementadas por instrumentos quantitativos e sustentados estatisticamente. Assim, na seção 3 deste artigo, realiza-se uma pesquisa sobre a Análise Envoltória de Dados (DEA - Data Envelopment Analysis) e Avaliação Cruzada (Cross Evaluation), 
a fim de contribuir para uma melhor compreensão de seu conceito e utilidade, voltando a aplicação dessas técnicas para a mobilidade urbana como uma forma alternativa de obtenção de índices e indicadores.

Diante do exposto, o objetivo central deste artigo é avaliar as eficiências das Regiões Administrativas do município do Rio de Janeiro calculadas a partir da Análise Envoltória de Dados e da Avaliação Cruzada, em função dos padrões de mobilidade considerados para esta análise, por meio do desenvolvimento de indicadores. Para isso, foi necessário não só compreender estas técnicas, como citado anteriormente, mas também entender como realizar os cálculos e melhor escolher as variáveis que envolvem o problema. 0 uso dessas ferramentas se justifica pela aplicabilidade em identificar e apontar a eficiência de unidades produtivas, com a flexibilidade de se considerar diferentes variáveis que podem ser testadas e analisadas a partir de estatísticas que respaldam a escolha dos indicadores e seus resultados. Além do mais, constatou-se a existência de poucos estudos que as apliquem à mobilidade urbana, fato verificado neste artigo por meio de um levantamento feito nas bases de pesquisa, como será melhor descrito nos itens subsequentes, o que justifica divulgá-las e realçar o potencial das mesmas, estimulando seu uso.

A partir desta introdução, este artigo é estruturado em 7 seções. A seção 2 aborda a mobilidade sustentável e indicadores. A seção 3 traz o conceito da Análise Envoltória de Dados e da Avaliação Cruzada. A seção 4 discorre sobre o município do Rio de Janeiro. A seção 5 explica a metodologia adotada neste artigo. A seção 6 apresenta os resultados obtidos para os dois indicadores desenvolvidos. Por fim, na seção 7, têm-se as considerações finais.

\section{MOBILIDADE SUSTENTÁVEL E INDICADORES}

0 grande volume de problemas decorrentes ou relacionados aos deslocamentos urbanos, tais como acidentes, congestionamentos, poluição sonora e atmosférica, tem se tornado bastante expressivo nos dias atuais. Como aponta Da Silva, Costa e Ramos (2010), há uma percepção crescente e generalizada de que é preciso buscar estratégias que viabilizem padrões mais sustentáveis de mobilidade. No anseio pelo desenvolvimento sustentável e tendo em vista as adversidades mencionadas, muito se investiga a respeito das formas de melhor compreender e planejar o meio urbano.

O relatório da Comissão Brundtland, de 1987, define o desenvolvimento sustentável como “a satisfação das necessidades do presente sem comprometer a capacidade de as gerações futuras satisfazerem as suas próprias necessidades" (WCED, 1987). Embora seja referido inicialmente aos impactos ambientais, o conceito de sustentabilidade foi expandido para buscar um equilíbrio entre as qualidades ambientais, sociais e econômicas. Alguns autores como Seabra, Gonzales Taco e Dominguez (2013) e VTPI (2015) dissertam que não há um consenso sobre a definição de sustentabilidade, pois a mesma evolui com o tempo. No entanto, o conceito essencial, contido no Relatório de Brundtland, é amplamente aceito e utilizado, sendo, portanto, o adotado neste trabalho.

A mobilidade é um item essencial para o desenvolvimento urbano sustentável. Para que o planejamento e o gerenciamento da mobilidade aconteçam de forma adequada, é necessária a sua mensuração. Em geral, o desenvolvimento e a mobilidade sustentáveis são mensurados por meio de indicadores e/ou índices, concebidos e utilizados por instituições de pesquisa e pela administração pública, responsáveis pelo planejamento urbano e de transportes. Portanto, os governos devem formular e implementar políticas, com a finalidade de assegurar que os bene- 
fícios do crescimento urbano sejam compartilhados de forma democrática, equitativa e sustentável (ONU, 2014).

A mobilidade está relacionada com os deslocamentos diários de determinada população no seu espaço - não apenas sua efetiva ocorrência, mas também a possibilidade ou facilidade de ocorrência destas viagens. De acordo com Portugal (2017), as escolhas, envolvidas na programação das viagens, produzem padrões de mobilidade que são influenciados não só pelo transporte, mas também pelo uso do solo e pelas características socioeconômicas dos indivíduos. 0 transporte, com suas infraestruturas e serviços, facilita acessos que, junto ao uso do solo, promove as condições de acessibilidade. Ou seja, a mobilidade resulta também da interação entre as características individuais e as condições de acessibilidade, que dependem da integração entre transportes e uso do solo. Assim, no que tange ao planejamento urbano e de transportes, é importante entender a dinâmica de deslocamentos entre as regiões e enxergar como os usuários escolhem alternativas de transporte sustentável, o que envolve a inclusão do uso do solo e aponta sua importância.

Neste trabalho, pesquisa-se essa interação entre as regiões, tendo em vista que a localização de moradias e empregos (que será considerada uma variável) influencia os padrões de viagens (Waddell, 2011). O destaque ao "emprego" deve-se pela conhecida predominância das viagens urbanas com este propósito e nos horários de pico, o que se torna ainda mais crítico em cidades brasileiras, pela concentração excessiva desta atividade nas áreas mais centrais e por um acesso da periferia não baseado nas modalidades de maior capacidade e mais sustentáveis, como já comentado.

0 conceito de mobilidade sustentável é muito mais abrangente do que a mobilidade defendida pela abordagem tradicional, já que considera não só a atividade de transporte em si, mas as relações com os demais setores e atores da vida urbana (Gonzáles Villada e Portugal, 2015). Então, quando adicionado o conceito de sustentabilidade, se explicitam atributos qualitativos às viagens (não só os quantitativos), realçando a visão multimodal (e não apenas o tráfego motorizado) com base nas modalidades de menor impacto social e ambiental (Machado, 2010; Mello, 2015).

Costa (2008) aponta que os indicadores são variáveis selecionadas que podem ajudar a tornar os objetivos operacionais e reduzir a complexidade no gerenciamento de determinados sistemas. Quando os indicadores são referenciados a metas ou objetivos, eles tornam-se medidas de performance, revelando as condições de um sistema, organizações ou políticas (Gudmundsson, 2004). Para Maclaren (1996), indicadores são simplificações de fenômenos complexos, e podem revelar tendências históricas e prover informação indireta sobre o futuro de um sistema. Diante disso, a utilização, neste artigo, da Análise Envoltória de Dados (DEA) e Avaliação Cruzada, se mostra indicada em função de suas características citadas na seção 1 . Além de serem técnicas as quais não necessitam de especialistas para a escolha e determinação da importância das variáveis, elas resultam na estimativa de uma eficiência para cada região, permitindo uma comparação entre elas e, assim, fornecendo informações que podem influenciar diretamente as políticas de mobilidade.

Nesse sentido, com a aplicação da DEA, este trabalho elaborou dois indicadores de mobilidade sustentável, a fim de revelar a eficiência da mobilidade nas diferentes Regiões Administrativas do município do Rio de Janeiro, e que são apresentados no item 4. 


\section{ANÁLISE ENVOLTÓRIA DE DADOS E AVALIAÇÃO CRUZADA}

\subsection{Análise Envoltória de Dados}

A Análise Envoltória de Dados, desenvolvida por Charnes et al. (1994), é uma ferramenta matemática para a medida de eficiência de unidades produtivas. Essas unidades produtivas são denominadas de Decision Making Unit - DMU, ou Unidades Tomadoras de Decisão. Através da comparação entre as unidades, que possuem características operacionais similares, essa técnica fornece dados quantitativos sobre possíveis direções para melhorar o desempenho das unidades ineficientes. Para este trabalho, as DMU são as Regiões Administrativas do município do Rio de Janeiro.

Nos últimos anos, DEA tem sido utilizada para avaliar diversos contextos. Como alguns exemplos de utilização da DEA em serviços no Brasil, podem ser citados os trabalhos de Faria, Jannuzzi e Silva (2008), que analisaram a eficiência dos gastos municipais da cidade do Rio de Janeiro em saúde e educação. Já Calvo (2002), Marinho (2003) e Lobo (2010) realizaram estudos utilizando a DEA voltados para hospitais. Diante do exposto, realizou-se um levantamento bibliográfico a fim de encontrar artigos que analisem a eficiência da mobilidade urbana utilizando a DEA. Para isso, se realizou a pesquisa bibliográfica nas bases Web of Science, Science Direct e SciElo utilizando a combinação "DEA" e "urban mobility". Usou-se como critério de seleção, a escolha de títulos e resumos que englobem o assunto proposto. Ao final desta busca, obtiveram-se, ao todo, 17 artigos elaborados no período de 1993 e 2018. Após a filtragem, selecionaram-se 7 artigos que correlacionam mobilidade urbana e DEA, mostrando o quanto é singular essa aplicação.

Assim, compreender bem o conceito de eficiência é fundamental para esta análise, entendendo a sua natureza relativa entre as unidades produtivas (Mello et al., 2005). 0 objetivo primário da DEA consiste em comparar um certo número de DMU que realizam tarefas similares e se diferenciam nas quantidades dos recursos consumidos e das saídas produzidas. No entanto, a DEA tem como objetivo também identificar as DMU eficientes, medir e localizar a ineficiência e estimar uma função de produção linear por partes (piece-wise linear frontier), que fornece o benchmark (referência) para as DMU ineficientes (Antunes, Lins e Meza, 2000; Acosta, Da Silva e Lima, 2011). Assim, a eficiência é apresentada para fins deste trabalho como:

$$
\text { Eficiência }=\frac{\text { Output }}{\text { Input }}
$$

A equação 1 mostra que quanto maior o denominador (recursos disponíveis - input) para produzir o mesmo output (numerador), mais ineficiente é a unidade produtiva. Então, o intuito para que uma unidade produtiva se mantenha eficiente é preservar a maior distância (relação) entre o output e o input. Por exemplo, sob a perspectiva da mobilidade urbana, para o mesmo input, quanto maior o número das viagens geradas por transporte público e a quantidade de empregos em uma determinada região, ela tenderá a ser mais eficiente (ver seção 5).

Existem dois modelos que são considerados clássicos para determinação da fronteira na DEA, CCR e BCC. 0 modelo BCC, que é o utilizado neste artigo, por permitir que as variáveis das DMU operem sem ser favorecidas ou prejudicadas - o que está melhor explicado no item 5 também é conhecido como VRS - Variable Returns to Scale, devido a Banker et al. (1984). É um modelo matemático que considera retornos variáveis de escala, isto é, substitui o axioma da proporcionalidade entre inputs e outputs pelo axioma da convexidade (Giacomello e De Oliveira, 2014). Ao obrigar que a fronteira seja convexa, o modelo BCC permite que as DMU que operam com baixos valores de inputs tenham retornos crescentes de escala e as que operam com altos 
valores tenham retornos decrescentes de escala. O Modelo BCC utiliza, para cada DMU, o problema de programação linear apresentado na Figura 1.

A fronteira eficiente de produção (ou função fronteira de produção) representa a região do gráfico que é dada pela máxima quantidade de outputs (produtos) que podem ser obtidos a partir dos inputs (insumos) utilizados (Acosta, Da Silva e Lima, 2011; Antunes, Lins e Meza, 2000).

De acordo com Barba-Romero e Pomerol (1997), a DEA é usada na problemática da ordenação como ferramenta Multicritério de apoio à decisão. Dessa maneira, é um mecanismo que subsidia estratégias de produção que maximizam a eficiência das DMU avaliadas, e corrige as ineficientes por meio da determinação de alvos. Por conta disso que a DEA se mostra bastante útil e aplicável ao planejamento de transportes.

\begin{tabular}{|c|l|}
\hline \multicolumn{2}{|c|}{ Modelo BCC } \\
\hline Min $h_{0}$ & \multicolumn{1}{|c|}{} \\
Sujeito a & $h_{0}=$ eficiência da $\mathrm{DMU}_{0}$ em análise \\
$x_{i 0}=$ inputs da $\mathrm{DMU}$ \\
$h_{0} x_{i 0} \geq \sum_{k} \lambda_{k} \cdot x_{i k}, \forall_{i}$ \\
$y_{j 0}=$ outputs da $\mathrm{DMU}_{0}$ \\
$\sum_{k} \lambda_{k} \cdot y_{j k} \geq y_{j 0}, \forall_{j}$ & $\lambda_{k}=$ contribuição individual da $\mathrm{DMU}_{\mathrm{k}}$ \\
$\sum_{k}=$ contribuição individual da $\mathrm{DMU}_{\mathrm{k}}$ na definição do alvo da $\mathrm{DMU}_{0}$ \\
$\sum_{k} \lambda_{k}=1$ & $h_{0}$ e $y_{k}=$ variáveis de decisão \\
$y_{k} \geq 0, \forall_{k}$ & \\
\hline
\end{tabular}

Figura 1: Formulação do modelo BCC orientado a inputs

A figura 2 mostra a diferença entre a representação da eficiência na DMU nos modelos BCC e CCR. Ambos para orientação a inputs. No caso CCR, apenas a DMU representada pelo ponto B é considerada eficiente, enquanto no caso BCC, os pontos $\mathrm{A}, \mathrm{B}$ e $\mathrm{C}$ também são eficientes, pelo pressuposto de que há diferenças de desempenho em função da escala dos insumos (Ramos e Ferreira, 2007).

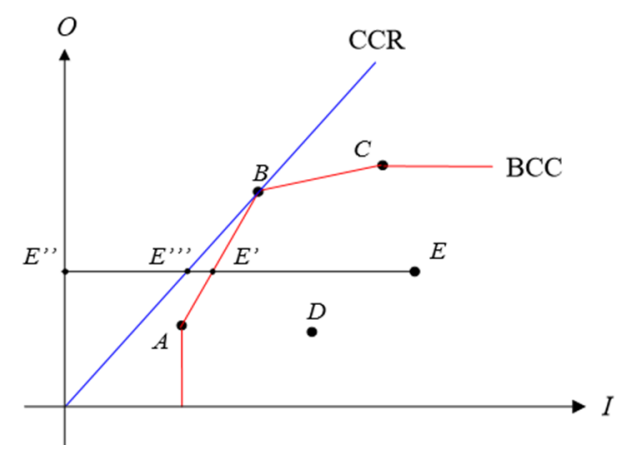

Figura 2: Gráfico de representação das fronteiras BCC e CCR

\subsection{Avaliação Cruzada}

De acordo com Antunes, Lins e Meza (2000), uma maneira de evitar as diferenças nos pesos determinados para cada DMU, sem a arbitrariedade das restrições e sem o conhecimento prévio da importância relativa de cada variável, é utilizando a Avaliação Cruzada. Ela foi desenvolvida por Sexton et al. (1986), e difere do modelo DEA padrão porque utiliza DEA em uma avaliação em conjunto em vez de uma auto-avaliação. Logo, a avaliação feita é uma média das eficiências de uma DMU calculadas sob o "ponto de vista" das outras DMU. 
Os modelos DEA clássicos atribuem peso aos insumos e produtos com objetivo de maximizar o índice de eficiência da DMU avaliada, sem violar determinadas restrições. Assim, o modelo atribui ponderações maiores aos pontos fortes da DMU avaliada, ou seja, aos outputs com maiores níveis de produção e aos inputs com menores níveis de consumo, enquanto aos inputs com elevados níveis de consumo e outputs com reduzidos níveis de produção são atribuídos pesos menores ou nulos. Consequentemente, o peso de cada variável insumo ou produto varia amplamente entre as DMU avaliadas. Tal desequilíbrio pode conduzir a esquemas de pesos inconsistentes com o conhecimento a priori acerca da importância relativa das variáveis insumos e produtos (Alcantara e Sant'Anna, 2002; Ramón et al., 2010; Ruiz e Sirvent, 2012).

A eficiência cruzada de uma DMU é calculada com base no esquema de pesos ótimos de uma DMU k é definida pelo seguinte quociente:

$$
\text { Eks }=\sum_{i}^{\text {outputs }} u_{i k} y_{\text {is }} / \sum_{j}^{\text {inputs }} V_{j k} X_{j s}
$$

Onde $u_{i k}$ e $v_{j k}$ são os pesos ótimos (soluções ótimas) da DMU k que ponderam, respectivamente, os produtos yis e os insumos $\mathrm{Xjs}_{\mathrm{s}}$ da DMU s.

A avaliação cruzada tem sido utilizada em diversos campos (Oral et al., 1991; Chen, 2002; Lu e Lo, 2007; Wu et al., 2009; Yu et al., 2010; Yang et al., 2012, 2013 apud Yu e Hou, 2016). No entanto, com base em pesquisa bibliográfica realizada nas bases Web of Science, Science Direct e SciElo utilizando a combinação "cross evaluation" e "urban mobility", não se obteve nenhum resultado de artigo abordando o assunto. Sendo assim, se verifica que, como o modelo DEA padrão, a avaliação cruzada também é pouco, ou quase nada, utilizada em trabalhos que abordem o tema "mobilidade urbana".

Nesse artigo, será aplicado o modelo BCC para a DEA, bem como será feita uma avaliação cruzada das DMU.

\section{4. ÁREA DE ESTUDO}

O presente trabalho aplica os métodos de DEA e da Avaliação Cruzada para avaliar a eficiência das Regiões Administrativas do município do Rio de Janeiro. De acordo com dados do Censo demográfico realizado em 2010 pelo IBGE, o município é dividido em 161 bairros, agregados em 33 Regiões Administrativas.

Nos anos 2003 e 2013, foram feitos para a região metropolitana do Rio de Janeiro, os Planos Diretores de Transportes Urbanos (PDTU), elaborados por meio de pesquisas domiciliares e de interpretação. Através desses relatórios é possível obter dados sobre o perfil dos usuários do sistema de transportes, bem como a matriz Origem-Destino, que tem como principal objetivo compreender os padrões de viagem dos residentes de determinada área e possibilitam ao setor técnico de transporte responder e trabalhar na melhora do sistema para a população (Bureau of Transport Statistics, 2013). O PDTU tem como função auxiliar o Governo do Estado do Rio de Janeiro a desenvolver políticas públicas setoriais, orientando as ações executivas relativas aos investimentos em infraestrutura viária, sistemas de transporte coletivo e terminais de integração, além de definir estratégias destinadas a otimizar a utilização da rede de transporte disponível, integrando e expandindo os sistemas existentes em redes futuras, de acordo com os objetivos firmados (CENTRAL, 2005).

Para a análise a ser desenvolvida, utilizaram-se dados da pesquisa Origem-Destino realizada pelos PDTU de 2003 e 2013, além de um valor referente à quantidade de emprego por região, 
extraído da Relação Anual de Informações Sociais-RAIS (MTE, 2013). Optou-se também por desconsiderar os dados de 9 das 33 Regiões Administrativas do município, devido ao baixo volume de entrevistas realizadas nesses locais. As 24 Regiões Administrativas analisadas são representadas em cinza no mapa da figura 3.



Figura 3: Regiões Administrativas do município do Rio de Janeiro.

\section{MATERIAIS E MÉTODOS}

Adotaram-se os métodos DEA e a Avaliação Cruzada para estimar as eficiências de cada uma das regiões definidas em função da mobilidade. Em um primeiro momento, utilizou-se o modelo de DEA padrão BCC para estimar a eficiência padrão de cada DMU. Em seguida, utilizou-se a Avaliação Cruzada, uma extensão da Análise Envoltória padrão, que calcula a eficiência de uma região sob o ponto de vista das outras (Antunes, Lins e Meza, 2000). Realizaram-se os cálculos pelo software SIAD.

Nos dados disponibilizados pela pesquisa domiciliar realizada para o PDTU, encontram-se variáveis referentes às características do usuário - idade, sexo, renda, grau de instrução e setor da atividade de trabalho - e variáveis referentes às características da principal viagem realizada no dia anterior, como origem, destino, motivo da viagem, hora de saída e chegada, modo de transporte e tempo de duração. Em concordância com o objetivo do trabalho, que deseja avaliar as regiões em função da mobilidade, as variáveis que se referem às características da viagem despertam maior interesse que às demais, pois elas expressam diretamente os padrões mobilidade, diferentemente de, por exemplo, variáveis socioeconômicas, que expressam características que influenciam esses padrões.

Já o emprego foi selecionado como variável de uso do solo, pois representa uma atividade realizada no local e a sua presença indica uma oportunidade a ser realizada pelos moradores, podendo refletir condições de acessibilidade. 0 dado fornecido pela RAIS se refere à quantidade de vagas de trabalho exercidas em uma determinada região, isto é, a quantidade de pessoas que trabalham nessa região, e não a quantidade de moradores que possuem emprego. Caso essa variável representasse a quantidade de pessoas que moram em uma região e que estão empregadas, ela seria melhor definida como uma variável socioeconômica. 
Sendo assim, a partir dos dados levantados, selecionaram-se cinco variáveis a serem utilizadas para o desenvolvimento de indicadores. São elas: o número de empregos (Emp), isto é, a quantidade de vagas de trabalho exercidas em cada região administrativa; a média das viagens geradas por transporte público (TP) e individual (TI) dentro de cada região; a razão entre essas viagens (TI/TP) e, por fim, o tempo médio despendido nas viagens (TVM) geradas por cada uma delas.

Desenvolveram-se dois indicadores para essa análise (figura 4). 0 primeiro utiliza a variável Transporte Público como output e as variáveis Transporte Individual e Tempo Médio de Viagem como input. Já o segundo indicador utiliza a variável Emprego como output e as variáveis Tempo Médio de Viagem e razão entre Transporte Individual e Público como input. Destaca-se o fato de que as variáveis de input e output dos indicadores expressam características das viagens (sejam variáveis de mobilidade ou acessibilidade), ao contrário de variáveis como Emissão de Poluentes Atmosféricos, por exemplo, que expressam consequências dos padrões de mobilidade. Além disso, é importante compreender que não há relação de causa e efeito entre as variáveis, já que a escolha delas é feita de forma que, dentro do contexto analisado (no nosso caso, a mobilidade) e segundo a metodologia de aplicação da DEA, quanto maior a variável de output e quanto menor a variável de input, mais alta será a eficiência da DMU em comparação com as demais. Na DEA não é necessário que as variáveis de input gerem efeitos nas de output, já que seu propósito é comparar as eficiências das DMU baseadas no desempenho dessas variáveis. A ferramenta não determina se a mobilidade de uma DMU é eficiente, mas sim expressa se, comparativamente a outras DMUs, essa mobilidade é mais ou menos eficiente, em função das variáveis testadas, contribuindo para melhor entender a relação entre elas e, consequentemente, entre a mobilidade e acessibilidade.

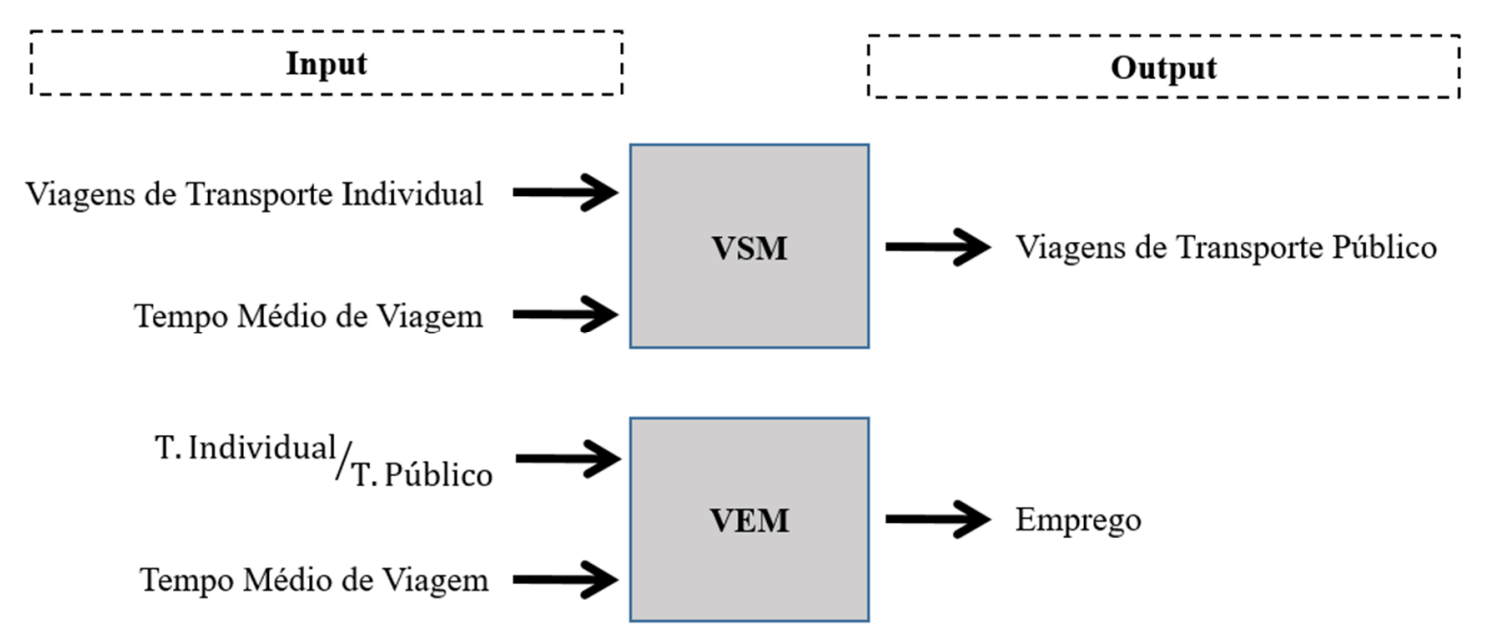

Figura 4: Indicadores elaborados.

A escolha das variáveis se justifica pelo caráter específico de cada indicador. Baseado na pesquisa desenvolvida por Litman (2015), é razoável constatar que a variável Transporte Público tem caráter social, tendo em vista que a disponibilidade de modos públicos e coletivos de transporte promove a acessibilidade de usuários de baixa renda, além de contribuir para a diversidade do sistema de transporte local. Dado que tal variável é definida como variável de output para o primeiro indicador, o mesmo é denominado Valor de Eficiência Social de Mobilidade (VSM). À variável Emprego pode-se atribuir caráter econômico, dado que seu valor evidencia a 
qualidade do desenvolvimento econômico e de negócios da região, bem como expressa a dimensão das oportunidades de trabalho, como já citado. Por isso, denomina-se o segundo indicador Valor de Eficiência Econômica de Mobilidade (VEM).

Essas variáveis foram utilizadas devido fundamentalmente a disponibilidade dos dados, mas ressalta-se que esta escolha conta com o respaldo da revisão bibliográfica que destaca, dentre as viagens motorizadas com foco na mobilidade sustentável, o papel favorável do transporte público e desfavorável do transporte individual. Por outro lado, nas cidades brasileiras, como o Rio de Janeiro, observam-se tempos significativos das viagens a trabalho em especial pela população das áreas periféricas dependentes dos núcleos centrais que concentram tal atividade. Portanto, tais variáveis incorporadas na modelagem estão sintonizadas não só com a bibliografia mas com a nossa realidade. No entanto, é recomendável que outras variáveis sejam contempladas em outros estudos de acordo com as especificidades locais.

Definidas as variáveis, a primeira etapa do processo de investigação dos dados constituiu na seleção das respostas originadas somente no município do Rio de Janeiro. Em seguida, agregaram-se as mesmas por Região Administrativa, como descrito no item anterior, e então gerou-se a Tabela 1 síntese com os valores a serem inseridos nos modelos.

Tabela 1: Variáveis inseridas na Análise Envoltória de Dados

\begin{tabular}{lllllll}
\hline Região Administrativa & Emp & TP & TI & TI/TP & TVM \\
\hline DMU1 & Bangu & 35.595 & 132.949 & 42.611 & 0,32 & 65 \\
DMU2 & Barra da Tijuca & 156.724 & 195.111 & 101.149 & 0,52 & 75 \\
DMU3 & Botafogo & 129.106 & 168.308 & 90.558 & 0,54 & 61 \\
DMU4 & Campo Grande & 58.677 & 157.646 & 56.806 & 0,36 & 79 \\
DMU5 & Centro & 333.206 & 323.584 & 95.653 & 0,30 & 58 \\
DMU6 & Copacabana & 49.029 & 116.243 & 59.284 & 0,51 & 63 \\
DMU7 & Ilha do Governador & 38.257 & 81.596 & 55.363 & 0,68 & 66 \\
DMU8 & Inhaúma & 23.121 & 61.547 & 25.053 & 0,41 & 57 \\
DMU9 & Irajá & 23.388 & 93.449 & 36.949 & 0,40 & 53 \\
DMU10 & Jacarepaguá & 104.666 & 229.030 & 111.821 & 0,49 & 67 \\
DMU11 & Lagoa & 77.686 & 168.291 & 97.886 & 0,58 & 67 \\
DMU12 & Madureira & 49.514 & 155.451 & 36.524 & 0,23 & 60 \\
DMU13 & Maré & 1.802 & 64.380 & 16.604 & 0,26 & 58 \\
DMU14 & Meier & 103.155 & 132.798 & 52.916 & 0,40 & 58 \\
DMU15 & Pavuna & 31.647 & 51.697 & 11.837 & 0,23 & 68 \\
DMU16 & Penha & 41.639 & 77.493 & 27.736 & 0,36 & 59 \\
DMU17 & Portuária & 30.076 & 44.509 & 15.632 & 0,35 & 58 \\
DMU18 & Ramos & 101.902 & 45.640 & 21.623 & 0,47 & 53 \\
DMU19 & Realengo & 19.432 & 44.007 & 20.812 & 0,47 & 61 \\
DMU20 & Santa Cruz & 31.666 & 80.370 & 29.844 & 0,37 & 100 \\
DMU21 & São Cristóvão & 64.610 & 50.503 & 16.143 & 0,32 & 56 \\
DMU22 & Tijuca & 82.429 & 86.971 & 40.480 & 0,47 & 58 \\
DMU23 & Vigário Geral & 30.706 & 34.729 & 8.237 & 0,24 & 60 \\
DMU24 & Vila Isabel & 37.299 & 131.255 & 49.299 & 0,38 & 63 \\
\hline Fonte: Elaboração própria a partir de PDTU (2003);PDTU(2013) e MTE (2013) & & & \\
\hline
\end{tabular}

Optou-se por aplicar o modelo BCC, pois, como explicado anteriormente, o mesmo considera retornos variáveis de escala, ou seja, permite que as variáveis das DMU que operam com baixos valores de inputs não tenham seus pesos reduzidos no cálculo (retornos crescentes de escala), enquanto que as que operam com altos valores não tenham pesos favorecidos nos cálculos (retornos decrescentes de escala). A eficiência das Regiões Administrativas do Rio de Janeiro é alterada pelo efeito de escala, pois é racional supor que o desempenho de cada região pode diferir das demais em função da escala de cada variável. 
No intuito de incrementar a análise, decidiu-se aplicar o método de Avaliação Cruzada, já que, por avaliar cada DMU a partir da média das eficiências individuais (denominada eficiência cruzada), o método diferencia mais claramente as regiões mais eficientes e permite estabelecer um ranking entre elas. Isso possibilita também a identificação das regiões administrativas que apresentam maior diferença entre as eficiências padrão e cruzada.

\section{RESULTADOS ENCONTRADOS}

A partir da aplicação dos modelos DEA-BCC e Avaliação Cruzada com os dados da Tabela 1, gerou-se a Tabela 2 e os gráficos apresentados nas Figura 5 e Figura 6.

As colunas VEM e VSM da Tabela 2 apresentam os resultados encontrados pelo programa para os dois indicadores. Para o melhor entendimento desses resultados, organizaram-se as variáveis utilizadas em tercis, isto é, as mesmas foram divididas em três classes, de igual quantidade de DMU e de acordo com sua grandeza. Por exemplo, as DMU enquadradas no primeiro tercil (T1) da variável Emprego, são as oito Regiões Administrativas com maior número de empregos, bem como as enquadradas no terceiro tercil (T3) são as que apresentaram o menor número de empregos. Lembrando que existe uma diferença entre os resultados de cada variável: no caso do Emprego, quanto mais alto o valor da variável, melhor para eficiência da DMU em comparação com as demais, ao contrário das variáveis Tempo Médio de Viagem e a razão TI/TP.

Tabela 2: Resultado da aplicação do DEA-BCC e Avaliação Cruzada

\begin{tabular}{|c|c|c|c|c|c|c|c|c|c|}
\hline \multirow{3}{*}{$\begin{array}{l}\text { Quadrante } \\
\text { (Avaliação Cruzada) }\end{array}$} & & & \multicolumn{2}{|c|}{ BCC } & \multicolumn{2}{|c|}{ A. Cruzada } & \multicolumn{3}{|c|}{ Tercil } \\
\hline & \multicolumn{2}{|c|}{ Região Administrativa } & VEM & VSM & VEM & VSM & Emp & TI/TP & TVM \\
\hline & DMU5 & Centro & 1,000 & 1,000 & 1,000 & 0,902 & T1 & $\mathrm{T} 1$ & T1 \\
\hline \multirow[t]{6}{*}{ QI } & DMU14 & Meier & 0,935 & 0,928 & 0,282 & 0,587 & T1 & $\mathrm{T} 2$ & T1 \\
\hline & DMU21 & São Cristóvão & 0,996 & 1,000 & 0,192 & 0,581 & $\mathrm{~T} 2$ & $\mathrm{~T} 1$ & T1 \\
\hline & DMU12 & Madureira & 1,000 & 1,000 & 0,156 & 0,931 & $\mathrm{~T} 2$ & $\mathrm{~T} 1$ & $\mathrm{~T} 2$ \\
\hline & DMU15 & Pavuna & 1,000 & 1,000 & 0,093 & 0,713 & T3 & $\mathrm{T} 1$ & T3 \\
\hline & DMU1 & Bangu & 0,883 & 0,879 & 0,096 & 0,692 & $\mathrm{~T} 2$ & $\mathrm{~T} 1$ & T3 \\
\hline & DMU13 & Maré & 1,000 & 1,000 & 0,006 & 0,718 & T3 & $\mathrm{T} 1$ & T1 \\
\hline \multirow[t]{8}{*}{ Q॥ } & DMU23 & Vigário Geral & 0,996 & 1,000 & 0,096 & 0,645 & T3 & $\mathrm{T} 1$ & $\mathrm{~T} 2$ \\
\hline & DMU4 & Campo Grande & 0,738 & 0,740 & 0,134 & 0,625 & $\mathrm{~T} 2$ & $\mathrm{~T} 2$ & T3 \\
\hline & DMU24 & Vila Isabel & 0,883 & 0,874 & 0,098 & 0,608 & $\mathrm{~T} 2$ & $\mathrm{~T} 2$ & $\mathrm{~T} 2$ \\
\hline & DMU16 & Penha & 0,941 & 0,942 & 0,116 & 0,581 & $\mathrm{~T} 2$ & $\mathrm{~T} 2$ & $\mathrm{~T} 2$ \\
\hline & DMU9 & Irajá & 1,000 & 1,000 & 0,068 & 0,567 & T3 & $\mathrm{T} 2$ & T1 \\
\hline & DMU11 & Lagoa & 0,796 & 0,813 & 0,173 & 0,430 & T1 & T3 & T3 \\
\hline & DMU6 & Copacabana & 0,844 & 0,848 & 0,118 & 0,461 & $\mathrm{~T} 2$ & T3 & $\mathrm{T} 2$ \\
\hline & DMU7 & Ilha do Governador & 0,798 & 0,796 & 0,083 & 0,340 & $\mathrm{~T} 2$ & T3 & T3 \\
\hline \multirow[t]{6}{*}{ Q III } & DMU19 & Realengo & 0,859 & 0,896 & 0,049 & 0,409 & T3 & T3 & $\mathrm{T} 2$ \\
\hline & DMU17 & Portuária & 0,946 & 0,970 & 0,084 & 0,519 & T3 & $\mathrm{T} 1$ & $\mathrm{~T} 2$ \\
\hline & DMU8 & Inhaúma & 0,934 & 0,953 & 0,063 & 0,503 & T3 & $\mathrm{T} 2$ & $\mathrm{~T} 1$ \\
\hline & DMU20 & Santa Cruz & 0,627 & 0,630 & 0,061 & 0,504 & T3 & $\mathrm{T} 2$ & T3 \\
\hline & DMU2 & Barra da Tijuca & 0,730 & 0,733 & 0,330 & 0,477 & T1 & T3 & T3 \\
\hline & DMU10 & Jacarepaguá & 0,802 & 0,829 & 0,242 & 0,520 & $\mathrm{~T} 1$ & T3 & T3 \\
\hline \multirow[t]{3}{*}{ QIV } & DMU22 & Tijuca & 0,924 & 0,912 & 0,216 & 0,482 & $\mathrm{~T} 1$ & $\mathrm{~T} 2$ & $\mathrm{~T} 1$ \\
\hline & DMU3 & Botafogo & 0,876 & 0,882 & 0,311 & 0,465 & $\mathrm{~T} 1$ & T3 & $\mathrm{T} 2$ \\
\hline & DMU18 & Ramos & 1,000 & 1,000 & 0,282 & 0,425 & $\mathrm{~T} 1$ & T3 & $\mathrm{T} 1$ \\
\hline
\end{tabular}

O gráfico da Figura 5 apresenta a dispersão dos resultados encontrados pelo modelo DEABCC para os indicadores VSM (eixo vertical) e VEM (eixo horizontal). 


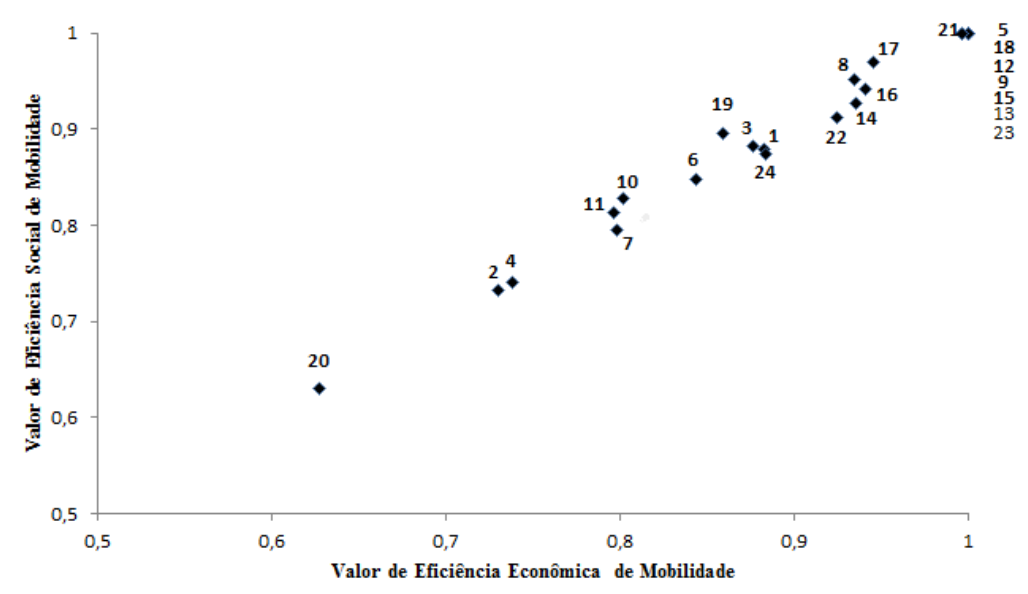

Figura 5: Resultado dos indicadores após aplicação do DEA-BCC

A DEA considera as Regiões Administrativas Maré (DMU13) e Vigário Geral (DMU23) “falsos positivos", isto é, as mesmas mostram alta eficiência e alta ineficiência simultaneamente, o que não corresponde a uma eficiência real (Serapião et al., 2003). Isso acontece quando a eficiência padrão e a eficiência invertida, a qual não é apresentada pela análise, atingem $100 \%$ de desempenho. Como esse artigo não trata da eficiência invertida, essa questão não será aprofundada e os resultados obtidos por essas DMU são desconsiderados da análise.

Aparentemente, os pontos se concentram na linha da Função Identidade, pois encontraramse valores aproximados nos dois indicadores para todas as DMU. Isso pode ter ocorrido devido a composição dos indicadores gerar a uma correlação entre eles: as variáveis TVM e TI são input nos dois indicadores e a variável TP está presente no denominador da fração no input de VEM e no output de VSM, ou seja, o comportamento dessas variáveis exerce a mesma influência no desempenho dos dois indicadores.

Outra possível interpretação para esse fenômeno é que, para as Regiões Administrativas do Rio de Janeiro, quando a mobilidade é economicamente eficiente, é também socialmente eficiente e vice-versa, lembrando que isso é válido quando considerado o efeito de escala. Essa relação de dependência entre os indicadores revela uma perspectiva interessante, tendo em vista o desenvolvimento de políticas públicas que visem elevar a eficiência de determinada região, pois no caso de investimentos na eficiência social (melhorando a variável Transporte Público por exemplo), é esperado um aumento consequentemente na eficiência econômica da região em função da mobilidade.

Constata-se pela Figura 5 que quanto mais perto do ponto de coordenadas $(1,1)$ do gráfico, maior a eficiência da região. Nesse caso, cinco Regiões Administrativas atingiram a eficiência máxima da mobilidade: Centro (DMU5), Ramos (DMU18), Madureira (DMU12), Irajá (DMU9) e Pavuna (DMU15). Se destaca o fato de que nas cinco Regiões Administrativas existe pelo menos uma estação metroferroviária. Os pontos referentes a elas encontram-se sobrepostos no ponto $(1,1)$ do gráfico.

Sobre a DMU5 (Centro), é interessante observar que, dentre as de maior eficiência, é a única que tem as três variáveis pertencentes ao tercil 1, ou seja, a mobilidade das demais regiões só alcançou a máxima eficiência porque as variáveis com baixo desempenho não tiveram peso significativo no cálculo da eficiência, ao passo que as variáveis com bom desempenho tiveram peso elevado. Isso ocorre devido ao efeito de escala. 
A DMU18 (Ramos) foi prejudicada pelo baixo desempenho da variável TI/TP devido à pouca utilização de transporte público na região em comparação com as demais, apesar da presença do transporte ferroviário. Esse é um fator importante na discussão acerca da baixa utilização desse modo em regiões periféricas do município do Rio de Janeiro, já que esta seria a opção mais indicada para viagens de grandes distâncias. Ainda assim, a DMU18 garante a alta eficiência da mobilidade por apresentar valores comparativamente elevados nas demais variáveis, tendo inclusive o segundo melhor desempenho na variável TVM.

Diferentemente da DMU18 (Ramos), a alta eficiência da mobilidade apresentada pela DMU12 (Madureira), que também conta com a presença do modo ferroviário, se deu pelo segundo melhor desempenho na variável TI/TP, relacionado à elevada utilização de modos de transporte público na região em comparação com as demais.

Já a DMU9 (Irajá), apesar do baixo número de empregos e desempenho médio na variável TI/TP, apresentou o melhor resultado para a variável TVM, garantindo a alta eficiência da mobilidade. A utilização tanto do transporte individual quanto do público nessa região está na média em relação às demais regiões, o que deve explicar o desempenho médio da variável TI/TP. Irajá é a única região com eficiência máxima da mobilidade com modo metroviário em vez de ferroviário.

Em comparação com as demais regiões, a DMU15 (Pavuna) obteve o melhor resultado para a variável TI/TP devido à baixa utilização do transporte individual nessa região, possivelmente pela renda relativamente menor de sua população, que pode afetar a posse e o uso do automóvel. 0 uso do transporte público também apresentou-se baixo, o que pode explicar o quarto pior desempenho no TVM.

A região com a mobilidade menos eficiente foi Santa Cruz (DMU20), cuja variável TVM é a pior entre todas as regiões, seguida de Barra da Tijuca (DMU2), com variáveis TVM e TI/TP muito baixas e Campo Grande (DMU4), a segundo pior TVM.

No gráfico referente à Avaliação Cruzada (Figura 6), as fronteiras desenhadas pelas médias dos indicadores delimitam a área de quatro quadrantes, o que também facilita a compreensão das diferenças entre as eficiências estimadas.

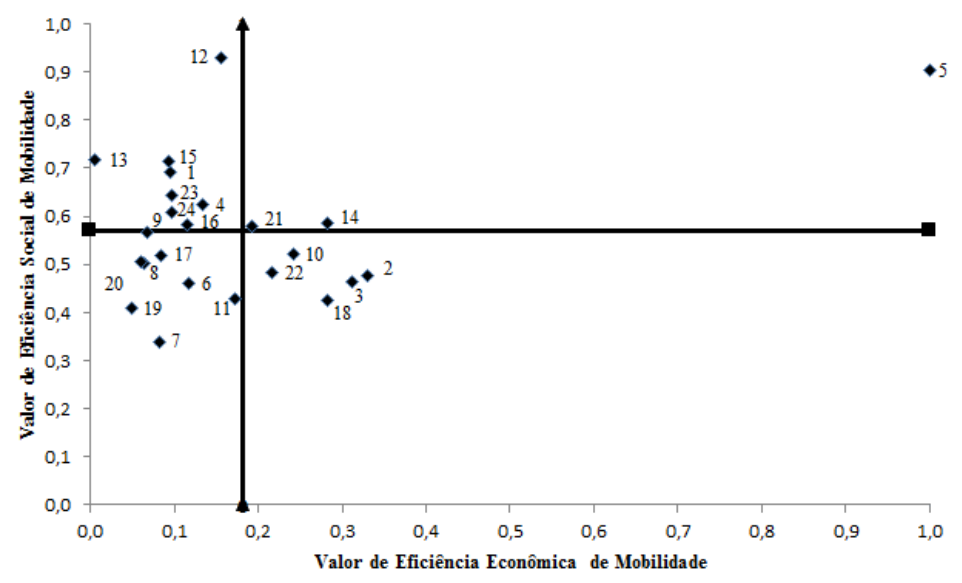

Figura 6: Resultado dos indicadores após aplicação da Avaliação Cruzada e suas médias

De antemão, é importante destacar que, diferentemente do ocorrido na DEA, na Avaliação Cruzada as DMU obtiveram resultados consideravelmente discrepantes para os dois indicadores. 
A análise de tercil pode indicar qual variável prejudica ou favorece a eficiência da DMU. Porém, é importante lembrar que esta é uma análise comparativa, ou seja, quando a mobilidade de uma determinada região é definida como eficiente, significa que ela é eficiente frente às demais regiões e de acordo com os indicadores propostos.

Em relação aos tercis correspondentes de cada variável, observam-se desempenhos semelhantes entre DMU localizadas no mesmo quadrante no gráfico. As Regiões Administrativas do Quadrante I, por exemplo, são mais eficientes que as demais nos dois indicadores e apresentam a maior parte das variáveis no tercil 1. As dos Quadrantes II e IV são eficientes em um dos indicadores apenas: no VSM e no VEM, respectivamente. Esse comportamento é esperado, pois a maior parte das DMU do QII tem a variável TI/TP no tercil 1 (variável usada para o VSM), enquanto a maior parte das DMU do QIV tem a variável Emprego no tercil 1 (variável usada para o VEM).

Por fim, as regiões do Quadrante III são as menos eficientes em comparação com as demais. Como esperado, mais da metade das variáveis dessas regiões pertencem ao tercil 3, isto é, apresentam valores ruins se comparados com as outras (baixo Emprego e elevado TI/TP e TVM). Destaca-se a Ilha do Governador (DMU7), Realengo (DMU19) e Santa Cruz (DMU20), as quais não apresentam variáveis no tercil 1 e são as menos eficientes. É interessante observar que cada uma dessas eficiências foi prejudicada por uma variável diferente. Destaca-se ainda o Centro (DMU5) com a melhor eficiência para os dois indicadores, assim como na DEA e única região com todas as variáveis no tercil 1.

\section{CONSIDERAÇÕES FINAIS}

Com base na revisão bibliográfica apresentada e com uso da Análise Envoltória de Dados e da Avaliação Cruzada, foi possível atingir o objetivo central do artigo de avaliar as eficiências das Regiões Administrativas do município do Rio de Janeiro em função dos padrões de mobilidade existentes em cada uma delas. Nesse processo utilizaram-se a DEA e a Avaliação Cruzada para a criação de indicadores no quesito mobilidade.

Os resultados apresentados se mostraram consistentes e fornecem informações relevantes no que diz respeito tanto ao planejamento de transportes, por subsidiar informações para tomadas de decisões na sua infraestrutura e serviços (implementando, por exemplo, uma rede estruturante de transporte público baseada nas modalidades de maior capacidade, mais equitativa, qualificada e integrada), quanto para o planejamento do uso do solo (promovendo, por exemplo, novas centralidades e uma distribuição mais equilibrada dos empregos). Portanto, estas técnicas permitem articular condições de acessibilidade (envolvendo a facilidade de alcançar atividades representada por indicadores de transporte e uso do solo) bem como a mobilidade expressa por padrões de viagens. Nesse sentido, contribuem na melhor compreensão das condições que influenciam na mobilidade nas regiões estudadas. Esse último fator, inclusive, atende ao que diz a Lei Federal no 12.587/2012 - "Lei da Mobilidade".

A título de exemplo, pode-se hierarquizar as regiões, identificar quais as mais e as menos eficientes e determinar as variáveis que influenciam nesse desempenho, permitindo elaborar estratégias para reverter este quadro, principalmente no que diz respeito as localidades mais críticas quanto a sua eficiência.

O artigo ainda apresenta uma abordagem alternativa por meio da utilização da DEA e Avaliação Cruzada no estudo da mobilidade urbana e pretende, com isso, incentivar novos trabalhos 
ao mostrar que é possível obter resultados plausíveis e coesos. Foi permitido identificar variáveis, criar indicadores e analisá-los por meio da eficiência das unidades produtivas. Entretanto, esta abordagem apenas complementa e agrega-se às existentes, dentre as quais destacam-se os modelos integrados revisados por Sousa et al. (2017), reconhecendo-se a complexidade e o desafio que envolve melhor entender a relação entre transporte e uso do solo, como que isto interfere na realização das atividades desejadas e na promoção de uma mobilidade e um desenvolvimento mais sustentáveis. Foi mostrado que a DEA e a Avaliação Cruzada apresentam resultados discrepantes entre si, devido às consideráveis diferenças de metodologia de cálculo. As regiões Portuária (DMU17) e Realengo (DMU19), por exemplo, apresentam, ainda que em diferente proporção, alta eficiência na primeira análise, quando considerado o efeito de escala e pouco eficiente na segunda, quando suas eficiências são comparadas com as demais regiões de forma conjunta. A região Centro (DMU5) se mostrou eficiente nas duas abordagens, devido ao fato de apresentar as melhores variáveis, ao contrário de Santa Cruz, considerada uma das menos eficientes também nas duas abordagens.

A maior parte dos dados utilizados para compor as variáveis foram obtidos de pesquisas Origem-Destino realizadas há mais de cinco (2013) e dez (2003) anos, o que pode interferir na confiabilidade dos valores inseridos no modelo, com consequências nos resultados da análise e nas conclusões obtidas. A produção desse tipo de dados na escala do PDTU ainda é pouco valorizada no Brasil e por isso é provável que passe por alguns aprimoramentos, o que deverá gerar dados mais concisos e possibilitará novos ajustes. Além disso, novas estruturas de transporte foram instaladas no Rio de Janeiro nos últimos anos com foco nos eventos esportivos que ocorreram no município, como linhas de BRT e VLT e, por isso, uma nova análise com dados mais atualizados devem resultar em diferentes conclusões.

Por fim, como sugestão para futuros trabalhos, indica-se a utilização de outras variáveis de mobilidade urbana, como por exemplo o emprego per capita, indicadores de outras atividades (como número de matrículas escolares no caso do ensino) e variáveis de caráter socioeconômico, o desenvolvimento de indicadores de outras naturezas (como a ambiental), e a análise de outros dados resultantes da DEA, como a fronteira eficiente, fronteira invertida e benchmark.

\section{AGRADECIMENTOS}

Ao CNPq, Conselho Nacional de Desenvolvimento Científico e Tecnológico - Brasil, pelo apoio na realização deste trabalho.

\section{REFERÊNCIAS}

Acosta, C. M. M.; Azambuja, A. M. V.; Lima, M. L. P. de. (2011) Aplicação de Análise Envoltória de Dados (DEA) para medir a eficiência em portos brasileiros. Revista de Literatura de Transportes, São José do Campos, v. 5, n. 4, p. 88-102. DOI: 10.11606/d.18.2017.tde-25092017-112048

Alcantara, A. A. M.; Sant'Anna, A. P. (2002). Medindo Eficiência em Desenvolvimento de Sistemas. Produção, v.11, n; 2. DOI: $10.1590 / \mathrm{s} 0103-65132001000200006$

Antunes, C. H.; Lins, M. P. E.; Meza, L. A. (2000) Análise Envoltória de Dados e Perspectivas de Integração no Ambiente do Apoio à Decisão. Editora da COPPE/UFRJ, Rio de Janeiro.

Banker, R.D.; Charnes, A.; Cooper, W.W. (1984) Some models for estimating technical scale inefficiencies in data envelopment analysis. Management Science, v. 30, n. 9, p. 1078-1092. DOI: 10.1287/mnsc.30.9.1078

Barba-Romero, S.; Pomerol, J.C. (1997) Decisiones multicriterio: fundamentos teóricos e utilización práctica. Colección de Economia de Universidad de Alcalá, Madrid.

Bertolini, L. (2017) Planning the mobile metropolis - Transport for People, Places and the Planet. Macmillan education Palgrave, 253 p. DOI: 10.1080/01441647.2017.1355856

Bureau of Transport Statistics. (2013) Household Travel Survey report: Sydney 2012/13. Sydney.

Calvo, M. C. M. (2002) Hospitais públicos e privados no Sistema Único de Saúde do Brasil: o mito da eficiência privada no estado de Mato Grasso em 1998. 223 p. Tese (Doutorado em Engenharia de Produção). Universidade Federal de Santa Catarina, Florianópolis/SC.

Censo Demográfico 2010. Informações dos Bairros e Regiões Administrativas. IBGE, 2011. Disponível em: <http://downloads.ibge.gov.br/downloads_estatisticas.htm>. Acesso em 20 de março de 2017. 
Central (2005) Plano Diretor de Transporte Urbano da Região Metropolitana do Estado do Rio de Janeiro: resultado da pesquisa Origem/Destino. Rio de Janeiro.

Cervero, R. B. (2013) Linking Urban Transport and Land Use in Developing Countries. Journal of Transport and Land Use. Mineapolis, Jan., p. 7-24. DOI: 10.5198/jtlu.v6i1.425

Charnes, A.; Cooper, W. W.; Rhodes, E. (1978) Measuring the efficiency of decision making units.

European Journal of Operational Research, v. 2, p. 429-444. DOI: 10.1016/0377-2217(78)90138-8

Costa, M. (2008) Um índice de mobilidade urbana sustentável. Tese (Doutorado em Engenharia Civil). Escola de Engenharia de São Carlos . Universidade de São Paulo, São Carlos, SP. DOI: 10.11606/T.18.2008.tde-01112008-200521

Da Silva, A. N. R.; Costa, M. S.; Ramos, R. A. R. (2010). Development and application of I_SUM - An index of Sustainable Urban Mobility. Anais do 89th Transportation Research Board, TRB, Washington, D.C. DOI: 10.1.1.1008.9281

Faria, F. P.; Jannuzzi, P. M.; Silva, S. J. (2008) Eficiência dos gastos municipais em saúde e educação: uma investigação através da análise envoltória no estado do Rio de Janeiro. Revista de Administração Pública, v. 42, n. 1, p. 155-177. DOI: $10.1590 / \mathrm{s} 0034-76122008000100008$

Giacomello, C. P.; De Oliveira, R. L. (2014). Análise Envoltória de Dados (DEA): uma proposta para avaliação de desempenho de unidades acadêmicas de uma universidade. Revista Gestão Universitária na América Latina - GUAL, Florianópolis. v.7, n.2, p. 130-151. DOI: 10.5007/1983-4535.2014v7n2p130

Gonzáles Villada, C. A.; Portugal, L. S. (2015). Mobilidade Sustentável e o Desenvolvimento Orientado ao Transporte Sustentável. XXIX Congresso Nacional de Pesquisa em Transporte da ANPET, Ouro Preto.

González Villada, C. A. (2016) Procedimento Metodológico para a aplicação do TOD em países em desenvolvimento. Dissertação de Mestrado. Programa de Engenharia de Transportes. COPPE. Universidade Federal do Rio de Janeiro, Rio de Janeiro, 165 p.

Gudmundsson, H. (2004). Sustainable Transport and Performance Indicators. In: Heste, R.E. \& Harrison, R.M. (Eds). Issues in Evironmental Science and Technology, n. 20, p. 35-63.

Jones, P. (2014) The evolution of urban mobility: The interplay of academic and policy perspectives. IATSS Research. V. 38, 1, p 7-13. DOI: 10.1016/j.iatssr.2014.06.001

Litman, T. A. (2015). Developing indicators for sustainable and livable transport planning. Victoria Transport Policy Institute.

Hull, A.; Silva, C.; Bertolini, L. (2012) Accessibility Instruments for Planning Practice. COST Office. 370 p. ISBN13: 978-989-203187-3.

Lins, M. P. E.; Meza, L. A. (2000) Análise envoltória de dados e perspectivas de integração no ambiente do Apoio à Decisão. Editora COPPE UFRJ, Rio de Janeiro.

Lobo, M. S. C. (2010). Aplicação da análise envoltória de dados (DEA) para apoio às políticas públicas de saúde: o caso dos hospitais de ensino. 2010. 228f. Tese (Doutorado em Engenharia de Produção). Universidade Federal do Rio de Janeiro, Rio de Janeiro, RJ.

Machado, L. (2010). Índice de mobilidade sustentável para avaliar a qualidade de vida urbana. Dissertação (Mestrado em Planejamento Urbano). Universidade Federal do Rio Grande do Sul, Porto Alegre, RS.

Maclaren, V. W. (1996). Urban Sustainability Reporting. Journal of the Amercan Planning Association, Chicago, v.62, n.2, p.184202. DOI: $10.1080 / 01944369608975684$

Marinho, A. (2003). Avaliação da eficiência técnica nos serviços de saúde nos municípios do Estado do Rio de Janeiro. Revista Brasileira de Economia, v. 57, n. 3, Rio de Janeiro, RJ. DOI: 10.1590/s0034-71402003000300002

Mello, A.J. R. (2015) A acessibilidade ao emprego e sua relação com o a mobilidade e o desenvolvimento sustentáveis: o caso da região metropolitana do Rio de Janeiro. Tese (Doutorado em Engenharia de Transportes). Universidade Federal do Rio de Janeiro COPPE/UFRJ, Rio de Janeiro, RJ.

Mello, J.; Meza, L.; Gomes, E.; Neto, L. (2005), 'Curso de análise de envoltória de dados', XXXVII Simpósio Brasileiro de Pesquisa Operacional.

Mori, K.; Yamashita, T. (2015) Methodological framework of sustainability assessment in City Sustainability Index (CSI): A concept of constraint and maximisation indicators. Habitat International. v. 45, p. 10 - 14. DOI: 10.1016/j.habitatint.2014.06.013

MTE. (2013) Relação Anual de Informações Sociais - RAIS. Ministério do Trabalho e Emprego. Brasília, DF.

ONU. (2014) World urbanization prospects: the 2014 revision - highlights, Department of Economic and Social Affairs, Population Division, ONU.

PDTU. (2003) Plano Diretor de Transporte Urbano da Região Metropolitana do Rio de Janeiro. Secretaria de Estado de Transportes. Governo do Estado do Rio de Janeiro, Rio de Janeiro, RJ.

PDTU. (2013) Plano Diretor de Transporte Urbano da Região Metropolitana do Rio de Janeiro. Secretaria de Estado de Transportes. Governo do Estado do Rio de Janeiro, Rio de Janeiro, RJ.

Pedro, L.; Da Silva, M.A.V.; Portugal, L.S. (2017). Desenvolvimento e Mobilidade Sustentáveis. Transporte, Mobilidade e Desenvolvimento Urbano. Editora Elsevier, p. 19 a 38

Portugal, L. S. (2017) Transporte, Mobilidade e Desenvolvimento Urbano. Editora Elsevier. Rio de Janeiro. p. 360.

Portugal, L.S.; Flórez, J.; Silva, A.N.R. (2010). Rede de pesquisa em transportes: um instrumento de transformação e melhora da qualidade de vida. Transportes, v. 28, n. 1, p. 6 - 16. DOI: 10.14295/transportes.v18i1.395

Ramón, N., Ruiz, J. L.; Sirvent, I. (2010). On the choice of weights profiles in cross-efficiency evaluations. Europeans Journal of Operational Research, v. 207, p. 1564- 1572. DOI: 10.1016/j.ejor.2010.07.022 
Ramos, R. E. B.; Ferreira, G. M. F. (2007). Analisando Retornos de Escala Usando DEA: um Estudo em Instituições de Ensino Tecnológico no Brasil. Universidade Federal do Rio Grande do Norte, Natal-RN

Ruiz, J. L.; Sirvent, I. (2012). On the DEA total weight flexibility and the aggregation in cross-efficiency evaluations. European Journal of Operational Research, v. 223, p. 732-738. DOI: 10.1016/j.ejor.2012.06.011

Seabra, L.O.; Gonzales Taco, W.; Dominguez, E. M. (2013). Sustentabilidade em transportes: do conceito às políticas públicas de mobilidade urbana. Revista dos Transportes Públicos, ANTP, ano 35, p. 103-122.

Serapião, B. P.; Gomes, E. G.; Mello, J. C. C. B. S.; Meza, L. A.; Lins, M. P. E. (2003). Análise de envoltória de dados no estudo da eficiência e dos benchmarks para companhias aéreas brasileiras. Revista Pesquisa Operacional, v. 23, n. 2, Rio de Janeiro, RJ. DOI: $10.1590 / \mathrm{s} 0101-74382003000200005$

Sousa, F.; Loureiro, C.F.G.; Lopes. A. S. (2017) Representação do fenômeno urbano por meio de modelos integrados dos transportes e uso do solo: revisão da literatura e discussão conceitual. Transportes, v. 25, p. 96 - 108. DOI: 10.14295/transportes.v25i4.1319

Souza Júnior, J. N. C. de. (2010). Avaliação da eficiência dos portos utilizando análise envoltória de dados: estudo de caso dos portos da região nordeste do Brasil. Journal of Transport Literature, v.7, n.4, p.75-106. DOI: 10.1590/s223810312013000400005

Tagore, M.R.; Sikdar, P.K. (1995). A new accessibility measure accounting mobility parameters in 7th World Conference on Transport Research. The University of New South Wales, Sydney.

VTPI. (2015). Sustainable transportation and TDM: planning that balances economic, social and ecological objectives. TDM Encyclopedia. Victoria Transport Policy Institute. Disponível em: <http://www.vtpi.org/tdm/tdm67.htm>. Acesso em 13 de junho de 2017.

Waddell, P. A. (2011). Integrated Land Use and Transportation Planning and Modelling: Addressing Challenges in Research and Practice. Transport Reviews, v. 31, n. 2, p. 209-229, DOI: 10.1080/01441647.2010.525671

WCED (1987). Our Common Future (The Bruntland Report). United Nations, World Commission Environment and Development. Oxford: Oxford University Press.

Yu, Q.; Hou, F. (2016). A cross evaluation-based measure of super efficiency in DEA with interval data, Kybernetes, v. 45 n. 4 , p. 666-679. DOI: 10.1108/k-05-2014-0089Acosta, C. M. M.; Azambuja, A. M. V.; Lima, M. L. P. de. (2011) Aplicação de Análise Envoltória de Dados (DEA) para medir a eficiência em portos brasileiros. Revista de Literatura de Transportes, São José do Campos, v. 5, n. 4, p. 88-102. 